\title{
COVID-19 in Patients with Atopic Dermatitis Treated with Dupilumab: Three Cases and a Literature Review
}

\author{
Justyna Ceryn (iD) ${ }^{1, *}$ \\ Michał Niedźwiedź' ${ }^{\prime *}$ \\ Małgorzata Skibińska' \\ Magdalena Ciążyńska² \\ Aleksandra Lesiak' \\ Joanna Narbutt'
}

'Department of Dermatology, Pediatric Dermatology and Dermatological

Oncology, International Doctoral School of the Medical University of Lodz, Lodz, 9I-347, Poland; ${ }^{2}$ Department of Proliferative Diseases, Nicolaus

Copernicus Multidisciplinary Centre for Oncology and Traumatology, Lodz,

Poland

*These authors contributed equally to this work
Correspondence: Justyna Ceryn Department of Dermatology, Pediatric Dermatology and Oncology, Medical University of Lodz, gen. Karola Kniaziewicza 1/5, Lodz, 91347, Poland Email justyna.ceryn@stud.umed.lodz.pl

\begin{abstract}
There are limited clinical data on the impact of the SARS-CoV2 infection on patients with dermatological conditions treated with biologics. Dupilumab is a recombinant human $\mathrm{IgG}_{4}$ human monoclonal antibody that inhibits IL4 and IL13 signaling, and is used for moderate-severe atopic dermatitis treatment. We present three patients with atopic dermatitis (AD) treated with dupilumab who contracted COVID-19. In all patients, the infection had a mild course, and only in one, as documented by SCORAD, EASI, and DLQI scores, the condition of the skin deteriorated, and a prolonged positive PCR COVID-19 test was observed. The mechanism of dupilumab action and more evidence for IL13 importance in lung damage caused by SARS-CoV2 suggest a possible explanation for a mild-moderate course of the infection in treated AD patients. Based on current knowledge, there is evidence to continue dupilumab treatment in AD patients with mild-moderate COVID-19; however, careful assessment is needed for each patient.
\end{abstract}

Keywords: COVID-19, dupilumab, atopic dermatitis

\section{Introduction}

Dupilumab is the first targeted biological therapy approved worldwide for atopic dermatitis (AD) treatment. This fully human $\operatorname{IgG}_{4}$ monoclonal antibody is directed against IL4R $\alpha{ }^{1}$ Inhibition of IL4R $\alpha$ blocks the transmission of IL4 and IL13 signals, which are important in the inflammatory pathway mechanisms observed in AD. Described as an immunomodulatory medication, dupilumabis registered for patients with moderate--severe AD and is also approved for asthma and chronic rhinosinusitis with nasal polyposis treatment. ${ }^{1,2}$

Due to the worldwide SARS-CoV2 pandemic declared by the World Health Organization in March 2020, many problems arose in dermatological therapy, not only with COVID-19-positive patients' treatment at the time of the infection but also with maintenance of their chronic diseases, ${ }^{3-5}$ specifically concerns related to immunosuppressants and immunomodulatory medications and their possible impact on the COVID-19 course in treated patients, as well as consequences of stopping the treatment in patients with poorly controlled skin conditions. ${ }^{3,5}$ In March 2020, the European Task Force on Atopic Dermatitis published a statement on SARS$\mathrm{CoV} 2$ infection and $\mathrm{AD}$ that medications interfering mainly with type 2 inflammation, eg, dupilumab, that do not seem to increase the rate of viral infections may be preferable to treatments with immunosuppressants during the COVID-19 
pandemic. $^{3}$ The authors hoped that more clinical data would help to assess the risk:benefit ratio for these patients. Despite enormous efforts to share knowledge on COVID-19-related topics, our clinical data on dupilumab treatment in patients with $\mathrm{AD}$ and its impact on those who had caught SARS-CoV2 are still limited. We report three patients who contracted COVID-19 while being treated with dupilumab, including the course of the disease and $\mathrm{AD}$-assessment scores throughout the infection period. Current experience and theories regarding dupilumab treatment during the SARS-CoV2 pandemic are also provided, based on a literature review.

\section{Case Reports}

Due to the COVID-19pandemic, the dupilumab-treatment pathway was modified for patients with $\mathrm{AD}$ at our department. Patients are seen once a month, with clinical assessments and laboratory tests if needed. One dose of dupilumab is administered on the day and a second provided to be injected by the patient at home 2 weeks later. A day before admission, all patients undergo a nasal swab for SARS-CoV2 PCR and are seen in person the next day only if the test is negative.

The first patient was a 27-year-old woman diagnosed with AD in early childhood. She also had a history of allergic rhinitis and conjunctivitis. She had previously been unsuccessfully treated with topical corticosteroids and oral cyclosporine. In August 2020, following a decision by her physician, she started dupilumab $200 \mathrm{mg}$ subcutaneously every 2 weeks after a loading dose of $400 \mathrm{mg}$ (her body weight was $55 \mathrm{~kg}$ ). Her baseline SCORAD (Scoring Atopic Dermatitis) was 50, EASI (Eczema Area and Severity Index) 22.8, and DLQI (Dermatology Life Quality Index) 17. After 1 month of treatment, SCORAD, EASI, and DLQI scores had significantly decreased $(16.5,1.8$, and 3 , respectively). Three months after starting the treatment, before the seventh dose of dupilumab, the patient had a positive SARSCoV2 PCR test. She was unable to attend her appointment and collect her medication, so the dose of dupilumab was postponed by 2 weeks. The infection had a mild course, with fatigue and loss of taste and smell lasting for 5 days. She did not notice any deterioration in skin lesions. Two weeks from the positive test, her SCORAD was 15.5, EASI 1, and DLQI 5.

The next patient was a 19 -year-old man with a 6-year history of $\mathrm{AD}$. His previous treatments included topical corticosteroids, phototherapy (narrow-band UVB), and oral cyclosporine. He also suffered from asthma and allergic rhinitis. His baseline assessments revealed scores of SCORAD 67, EASI 37.9, and DLQI 12. He started dupilumab $300 \mathrm{mg}$ subcutaneously every 2 weeks after a loading dose of $600 \mathrm{mg}$. After 1 month of therapy, significant clinical improvement was observed (SCORAD 40.4, EASI 18.1, DLQI 1). On the day of his seventh dose, a false-negative SARS-CoV2 test due to a laboratory error was found, and he was seen in the department (SCORAD 11.8, EASI 2.9, DLQI 6). The error was corrected the same day after he had had the medication injected. Ultimately, he was SARS-CoV2-positive. His only symptom of the infection was loss of taste and smell, which lasted for 6 days. He reported mild deterioration in skin lesions on his hands only, with some improvement in other areas. He continued dupilumab treatment without any break. An exacerbation in SCORAD (15.8) but not EASI (2.2) or DLQI (1) scores was observed 1 month later. There was no break in his dupilumab injection schedule.

Our third patient was a 17-year-old woman with severe AD since the age of 5 years. She had previously been unsuccessfully treated with topical corticosteroids, phototherapy (narrow-band UVB), oral cyclosporine, methotrexate, and systemic tralokinumab (anti-IL13, clinical trial). She also had a history of anxiety disorder treated with pregabalin. Her baseline results revealed SCORAD 99.4, EASI 72, and DLQI 20. She started dupilumab $300 \mathrm{mg}$ subcutaneously every 2 weeks after a loading dose of $600 \mathrm{mg}$. Before the fifth dose of dupilumab, similarly to the second patient, due to a laboratory error on that day, a false-negative SARS-CoV2 PCR test was reported, and she was able to collect her medication for another month. The error was corrected the same day after she had had the medication injected. She reported fatigue and a runny nose during the infection, with the symptoms lasting 10 days. She did, however, observe exacerbation of her skin lesions, with more erythema, papules, and scaliness on lower extremities, shoulders, and back. Her SCORAD on the day of her COVID-19 diagnosis was 99, EASI 66, and DLQI 21. On follow-up after 1 month (3 weeks later, she was still SARS-CoV2-positive), her results were SCORAD 78.3, EASI 45.5, and DLQI 18. One dose of her treatment was omitted during her infection following advice from the department. As her PCR test continued to be positive 3 weeks after initial diagnosis, she was finally cleared for attending the appointment by the infectious disease specialist. 
All three patients' scores on SCORAD, EASI, and DLQI are presented in Table 1.

\section{Discussion}

Management of chronic skin diseases during the COVID-19 pandemic has become a worldwide problem. Firstly, seeing patients in face-to-face appointments has became impossible in many regions and countries, due to the high numbers of infections and overloaded health systems. Secondly, many patients are afraid of attending appointments and starting or continuing systemic therapy, including biologics. ${ }^{3-5}$ Teledermatology services have developed very quickly in a majority of dermatology departments, with good effectiveness and high satisfaction scores from the patients. ${ }^{5-7}$ With more data available, recommendations and guidelines have been published to help advise dermatological patients regarding their treatments. ${ }^{3-5,8,9}$

Until recently, only isolated clinical case reports of patients with $\mathrm{AD}$ treated with dupilumab at the time of COVID-19 infection were available, with clinical details presented in Table 2. ${ }^{10-14}$ A large group of AD patients on systemic treatments was monitored by Chiricozzi et $\mathrm{al}^{5}$ during the first wave of COVID-19 in Italy in 2020. Interestingly, a majority of those patients were treated with dupilumab in mono- or combined therapy. During the observational period, 16 patients $(0.9 \%)$, of which 15 were treated with dupilumab, were diagnosed with COVID-19. Only three patients $(0.2 \%)$ needed hospitalization, and there were no deaths from COVID-19-related disease during this time. Due to the strict lockdown measures, data on precise skin condition of presenting patients around the time of COVID-19 based on SCORAD and EASI were not possible to include in featured reports. ${ }^{5,10-14}$ In our patients, all scores were available, including at the time before dupilumab was started, after 1 month, and within 2 weeks of COVID-19 diagnosis. Only in one of three patients was a significant deterioration in SCORAD, EASI, and DLQI observed on the day of infection diagnosis, with all parameters having improved 1 month later.

All reported cases showed similarity in the course of COVID-19 in patients treated with dupilumab. A majority of them had asymptomatic to mild courses of the infection, with few patients needing hospitalization. To the best of our knowledge, this study is the first to objectively show the dynamics of skin condition in $\mathrm{AD}$ patients treated with dupilumab who have contracted COVID-19.

No increased risk of COVID-19 has been observed among AD patients on dupilumab in northern Italy, a highepidemic area, during the first wave of the infection. ${ }^{15}$ Interestingly, $5.6 \%$ of patients in a group presented by Rossi et $\mathrm{al}^{12}$ decided to stop dupilumab treatment entirely, while another $8.4 \%$ stopped temporarily due to quarantine or COVID-19 symptoms. Napolitano et al ${ }^{16}$ observed 200 patients with $\mathrm{AD}$ on dupilumab, with $96.5 \%$ of them continuing treatment online or through telephone followup. None of the patients reported any worsening in skin symptoms. Chiricozzi et $\mathrm{al}^{5}$ reported that $9.9 \%$ of patients in their group stopped dupilumab at least temporarily, while in Georgakopoulos and Yeung's ${ }^{17}$ cohort of patients

Table I Patient scores of SCORAD, EASI, and DLQI before, during, and after COVID-I9 diagnosis

\begin{tabular}{|c|c|c|c|c|}
\hline & Follow-up & SCORAD & EASI & DLQI \\
\hline \multirow[t]{4}{*}{ Patient I } & Aug 3, 2020 & 50.0 & 22.8 & 17 \\
\hline & Sep 8, 2020 & 16.5 & 1.8 & 3 \\
\hline & Nov 12, 2020, COVID ${ }^{+\dagger}$ & - & - & 一 \\
\hline & Nov 24, 2020 & 15.5 & 1.0 & 5 \\
\hline \multirow[t]{4}{*}{ Patient 2} & Aug II, 2020 & 67.0 & 37.9 & 12 \\
\hline & Sep I, 2020 & 40.4 & 18.1 & I \\
\hline & Oct 29,2020, COVID $^{+\dagger}$ & 11.8 & 2.9 & 6 \\
\hline & Dec I, 2020 & 15.8 & 2.2 & I \\
\hline \multirow[t]{5}{*}{ Patient 3} & Sep 8, 2020 & 99.4 & 72.0 & 20 \\
\hline & Sep 22, 2020 & 88.7 & 64.4 & 17 \\
\hline & Oct $27 \& 29,2020$, COVID $^{+\dagger}$ & 99.0 & 66.0 & 21 \\
\hline & Nov 12 \& 16, 2020, COVID \pm ; Nov 17, 2020, COVID $+\dagger$ & - & - & - \\
\hline & Nov 27, 2020 & 78.3 & 45.5 & 18 \\
\hline
\end{tabular}

Notes: ${ }^{\dagger}$ Entries in bold indicate dates of positive SARS-CoV2 PCR tests.

Abbreviations: SCORAD, Scoring Atopic Dermatitis; EASI, Eczema Area And Severity Index; DLQI, Dermatology Life Quality Index. 
Table 2 Case-report data on patients with atopic dermatitis treated with dupilumab who contracted COVID-I9

\begin{tabular}{|c|c|c|c|c|c|c|c|c|}
\hline Authors & $\begin{array}{l}\text { Age, } \\
\text { years }\end{array}$ & Sex & Comorbidities & $\begin{array}{l}\text { COVID-19 } \\
\text { course }\end{array}$ & $\begin{array}{l}\text { Hospitalization } \\
\text { required }\end{array}$ & $\begin{array}{c}\text { Dupilumab } \\
\text { continuation } \\
\text { during } \\
\text { infection }\end{array}$ & $\begin{array}{c}\text { AD } \\
\text { deterioration } \\
\text { during } \\
\text { COVID-19 }\end{array}$ & $\begin{array}{c}\text { AD scores } \\
\text { availability } \\
\text { during COVID- } \\
19\end{array}$ \\
\hline $\begin{array}{l}\text { Ferrucci } \\
\text { et } \mathrm{al}^{10}\end{array}$ & 40 & $M$ & No data & Mild & No data & Continued & No data & Unavailable \\
\hline $\begin{array}{l}\text { Caroppo } \\
\text { et } \mathrm{al}^{\prime \prime}\end{array}$ & $\begin{array}{l}56 \\
72\end{array}$ & $\begin{array}{l}F \\
M\end{array}$ & $\begin{array}{l}\text { No data } \\
\text { No data }\end{array}$ & $\begin{array}{c}\text { Mild } \\
\text { Asymptomatic }\end{array}$ & $\begin{array}{l}\text { Yes } \\
\text { No }\end{array}$ & $\begin{array}{l}\text { Continued } \\
\text { No data }\end{array}$ & $\begin{array}{l}\text { No } \\
\text { No }\end{array}$ & $\begin{array}{l}\text { Unavailable } \\
\text { Unavailable }\end{array}$ \\
\hline \multirow[t]{2}{*}{$\begin{array}{l}\text { Rossi } \\
\text { et } \mathrm{al}^{12}\end{array}$} & 52 & $\mathrm{~F}$ & $\begin{array}{c}\text { No } \\
\text { comorbidities }\end{array}$ & Mild & No data & Postponed & No data & Unavailable \\
\hline & 53 & $\mathrm{~F}$ & $\begin{array}{l}\text { Severe obesity, } \\
\text { hypertension, } \\
\text { asthma, } \\
\text { depressive } \\
\text { disorder }\end{array}$ & Severe & Yes & Postponed & No data & Unavailable \\
\hline $\begin{array}{l}\text { Ordóñez- } \\
\text { Rubiano } \\
\text { et al }\end{array}$ & 22 & $M$ & $\begin{array}{c}\text { No } \\
\text { comorbidities }\end{array}$ & Asymptomatic & No data & Continued & No data & Unavailable \\
\hline $\begin{array}{l}\text { Ordóñez- } \\
\text { Rubiano } \\
\text { et al }\end{array}$ & 27 & $M$ & $\begin{array}{c}\text { HIV } \\
\text { allergic rhinitis }\end{array}$ & Mild & No & Continued & No & Partly available \\
\hline
\end{tabular}

Abbreviations: $M$, male; $F$, female; $A D$, atopic dermatitis.

treated with dupilumab for $\mathrm{AD}$, only $0.62 \%$ temporarily stopped treatment. These differences could be related to the point in time of the COVID-19 pandemic, as in due course more knowledge about the infection was gained and patients felt more reassured to continue with their treatment. Ordóñez-Rubiano et $\mathrm{al}^{13}$ presented a protocol for dupilumab-treated patients with $\mathrm{AD}$ that suggested that treatment should be stopped only in symptomatic COVID19 patients requiring intensive care. Our patients, who were young and without risk factors of severe COVID19 , had a mild disease course. One missed one dose, due to the COVID-19 diagnosis and being unable to collect the medication. The second omitted a dose later, due to the prolonged positive PCR results.

Interestingly, a 72-year-old man with severe $\mathrm{AD}$ on dupilumab reported by Caroppo et $\mathrm{al}^{11}$ also had positive SARS-CoV2 PCR results for three consecutive tests over 9 weeks. During this time, despite the risk factors of COVID-19 (ie, age over 65 years and male sex), the infection remained asymptomatic, with good general and skin condition. The authors did not specify whether the patient was taking dupilumab on schedule, despite being isolated. In two patients with asthma, persistent positive PCR tests for SARS-CoV2 were reported, which was also observed in one of our patients. ${ }^{18,19}$ Following opinion obtained from an infectious disease specialist, we stopped performing PCR tests in that patient, as otherwise she would have not been able to receive continued dupilumab treatment. Cento et $\mathrm{al}^{20}$ showed that prolonged positive PCR tests in COVID-19 patients were relatively common. They retrospectively analyzed 7,608 hospitalized patients who had recovered from COVID-19 and underwent follow-up PCR tests. Persistent positive results were present in $13.7 \%$ of 7,127 patients at 14 days and in $14.7 \%$ of 191 patients tested further between 40 and 60 days after hospital discharge. Bhalla et $\mathrm{al}^{19}$ suggested that the prolonged viral detection could have been caused by dupilumab influencing SARS-CoV2 antibody levels, as they observed this in an asthma patient treated with dupilumab and steroids, but not in asthma patients treated only with inhaled steroids. More data are clearly needed to clarify this, as it is difficult to compare results with only single patients with $\mathrm{AD}$ or asthma and prolonged PCR-positive tests on dupilumab reported. 
Some clinical data are available on dupilumab treatment of asthma and severe chronic rhinosinusitis with nasal polyps and concomitant SARS-CoV2 infection. ${ }^{18,19,21,22}$ While isolated clinical reports have described positive outcomes, Eger et al ${ }^{21}$ were less optimistic. They assessed the incidence and course of COVID19 in a Dutch population (Dutch Severe Asthma Registry RAPSODI) in patients with severe asthma on biologic therapies, including dupilumab, mepolizumab, reslizumab, and benralizumab. Patient details during the course of the infection were compared to data from the general Dutch population. The results revealed odds of hospitalization and intubation of 14 and 41 times, respectively, those patients with severe asthma on biologics. Those results, however, were not divided based on the biologic the patients were on, and data comparing the asthma population during COVID-19 with asthma patients on biologics were not available. The authors also noticed that comorbidities of patients with severe asthma might have been significantly different from the general population, which further limits the results. Nevertheless, as there is some evidence that dupilumab treatment can be potentially beneficial during the COVID-19 course, careful assessment is needed depending on the individual patient's circumstances and the disease treated.

\section{Dupilumab Mechanism of Action and COVID: What Do We Know? Dupilumab and Risk of Infection}

Risk of infection during dupilumab treatment in $\mathrm{AD}$ patients has been carefully assessed in clinical trials. In SOLO 1 and SOLO 2, overall infections rates were reported in $28 \%-35 \%$ of patients treated with dupilumab and $28 \%-33 \%$ of those receiving placebo. ${ }^{23}$ Specifically, upper respiratory tract infections were reported in $2.62 \%$ $2.97 \%$ of dupilumab patients and $2.14 \%-2.25 \%$ of patients taking placebo. In the LIBERTY and CHRONOS clinical trials, upper respiratory tract infections were reported in $10 \%$ of patients in both placebo and treatment groups. ${ }^{24}$ Eichenfield et al's ${ }^{1}$ analysis of all clinical trial data concluded that dupilumab therapy was connected to a reduced risk of serious infections in AD patients and that treatment with registered dosing did not reveal statistically significant increases in overall infection rates when compared with placebo. Our patients were on scheduled doses, with one patient receiving a $200 \mathrm{mg}$ maintenance dose due to the decision of her physician in charge.
During the COVID-19 pandemic, another important question arises regarding the effectiveness of vaccinations in patients with $\mathrm{AD}$ treated with dupilumab. A possible lower response to vaccinations in $\mathrm{AD}$ children has been discussed and thought to be related to type 2 cytokines, mainly IL4 and IL13, involvement in AD inflammatory pathways. ${ }^{25,26}$ In Blauvelt et al, ${ }^{26}$ dupilumab did not affect response to the tetanus and meningococcal vaccines.

What can be expected in COVID patients who are undergoing dupilumab treatment? The SARS-CoV2 infection pathophysiology has not been entirely understood and seems to be heterogeneous. It is known that SARS-CoV2 induces differentiation of $T$ cells to subclasses, such as $T_{h} 1$ and $T_{h} 17$, subsequently inducing massive release of proinflammatory cytokines that may lead to a cytokine storm. ${ }^{27}$ This in turn may inhibit activation of cytotoxic $\mathrm{CD}^{+} \mathrm{T}$ cells. The virus may also stimulate secretion of type 2 cytokines, including IL4, IL5, IL10, and IL13. Increased levels of IL4, IL5, and IL10 were observed in fatal cases of the SARS outbreak of $2002-2003 .^{28}$ Moreover, IL4 and IL10 may suppress $T_{h} 1 / T_{h} 17$-induced inflammation. The complexity of the immunoresponses generated by the virus makes the assessment of the influence of individual factors like dupilumab on the course of the infection harder. ${ }^{27}$ Recently, a possible role of IL13 in COVID-19 was brought to light, with Donlan et $\mathrm{al}^{29}$ linking this cytokine with infection severity in vitro and in vivo. Previously, it had been proven that higher levels of IL6 and TNF $\alpha$ play a significant role in cytokine storms damaging lungs in COVID-19 patients, which resulted in biological medications targeting the cytokines being introduced to the patients' treatment. ${ }^{27,29}$

IL13 is involved in many processes, including recruitment of eosinophils and M2 macrophages to the lungs and activation of fibroblasts, which leads to fibrosis and collagen deposition. ${ }^{30}$ It has been confirmed that increased levels of IL13 in COVID-19 patients are related to the necessity for oxygen therapy via high-flow nasal cannula or mechanical ventilation treatment. ${ }^{29,31}$ Donlan et $\mathrm{al}^{29}$ also characterized the impact of SARS-CoV2 infection in a transgenic mouse model of COVID-19, revealing enhanced gene engagement in IL4 and IL13 signaling, including IL13 receptors, such as IL4r $\alpha$ and IL13ral. They used anti-IL13 in infected mice, resulting in reduced symptoms and better outcomes measured by clinical scores, weight loss, and mortality. As anti-IL13 did not change the viral load, the clinical improvement was likely caused by interrupting IL13-signaling pathways. Their hypothesis that taking dupilumab, which blocks IL13 and IL4 signaling, may be beneficial, at least in some patients 
with COVID-19, was confirmed by a retrospective analysis of two cohorts of patients infected with SARS-CoV2. Dupilumab use (any indication) was associated with a lower risk of hospitalization, mechanical ventilation, and death in those patients. A study of asthma patients from the Netherlands did not show such positive results; however, only patients with serious asthma were included and different biological treatments were not separated in the assessment. ${ }^{21}$

Nevertheless, it seems that dupilumab may at least potentially prevent the fatal course of the infection, which is consistent with the available literature. ${ }^{27,29}$

\section{Dupilumab Assessment During COVID-19}

Two of our patients did not miss a dose of dupilumab and received the drug despite being infected, due to a laboratory error. These patients did not notice any significant deterioration in their skin condition during or after the infection. One patient's AD deteriorated, but due to prolonged positive results on PCR COVID-19 tests, administration of dupilumab was delayed, as she was not able to attend her appointments as per hospital regulations.

Stopping dupilumab may cause AD exacerbation and impact patients' quality of life. ${ }^{27,32}$ It may also cause potential loss of response upon restarting dupilumab treatment, due to the possible formation of neutralizing antidrug antibodies (ADAs). ${ }^{33}$ The impact of ADA levels is still unknown in $\mathrm{AD}$ treatment with dupilumab, and their occurrence is not as distinct as for biologic drugs used for psoriasis. ${ }^{27,33,34}$ During the SOLO-CONTINUE dupilumab trial, ADAs occurred in $11.3 \%$ of the placebo group (after dupilumab treatment), $6.0 \%$ in the dupilumab-every-8-weeks group, $4.3 \%$ in the dupilumab-every-2-weeks group and $1.2 \%$ in the dupilumab-weekly group. ${ }^{33}$ This indicates that less frequent dupilumab admission leads to higher levels of ADAs and potentially could reduce response when the treatment is reintroduced.

All data lead to the conclusion that administration of dupilumab should not be discontinued due to COVID-19, and if SARS-CoV2 infection is suspected or proven in a patient already treated with dupilumab, continuation of the treatment needs to be assessed individually.

\section{Conclusion}

According to our experience and the analysis of the currently available data, we can assume that there is no higher risk of developing a severe course of COVID-19 in patients with moderate-severe $\mathrm{AD}$ treated with dupilumab. Our three patients with severe AD treated with dupilumab developed COVID-19 in the 12th week (two patients) and the eighth week (one patient) of therapy. They noticed only fatigue and loss of smell and taste. As for $\mathrm{AD}$, during that time they developed COVID-19 exacerbation of skin lesions, such as more intense pruritus, and greater erythema was observed in one. Despite clinical trials confirming the safety of dupilumab in terms of infections, we need more data on patients with COVID-19 treated with dupilumab, due to the complexity of the immunoresponse caused by the virus. Reporting registries and an anonymous patient-reported survey (Surveillance Epidemiology of Coronavirus (COVID-19) under Research Exclusion) have been created to gather all information regarding the influence of infection and vaccination on patients with $\mathrm{AD}$ and alopecia, regardless of the type of treatment, that will in time give us more valuable data on those patients. ${ }^{35}$

For now, during the pandemic, we should encourage our patients to continue with their treatment, get vaccinated, and strictly comply with the sanitary regime.

\section{Informed-Consent Statement}

Written informed consent was obtained from patients to publish this paper.

\section{Acknowledgment}

This research was funded by statutory activities of the Medical University of Lodz (503/5-064-04/503-01).

\section{Author Contributions}

All authors made a significant contribution to the work reported, whether in conception, study design, execution, acquisition of data, analysis, interpretation, or in all these areas, took part in drafting, revising, or critically reviewing the article, gave final approval to the version to be published, have agreed on the journal to which the article has been submitted; and agree to be accountable for all aspects of the work.

\section{Disclosure}

The authors report no conflicts of interest for this work.

\section{References}

1. Eichenfield LF, Bieber T, Beck LA, et al. Infections in dupilumab clinical trials in atopic dermatitis: a comprehensive pooled analysis. Am J Clin Dermatol. 2019;20(3):443-456. doi:10.1007/s40257-01900445-7 
2. Thangaraju P, Venkatesan N, Sudha TYS, Venkatesan S, Thangaraju E. Role of dupilumab in approved indications of COVID-19 patient: an efficacy-based nonsystematic critical analysis. SN Compr Clin Med. 2020;1-5. doi:10.1007/s42399-02000510-x

3. Thyssen JP, Vestergaard C, Barbarot S, et al. European Task Force on Atopic Dermatitis: position on vaccination of adult patients with atopic dermatitis against COVID-19 (SARS-CoV-2) being treated with systemic medication and biologics. $J$ Eur Acad Dermatol Venereol. 2021;35(5):e308-e311. doi:10.1111/jdv.17167

4. Ricardo JW, Lipner SR. Considerations for safety in the use of systemic medications for psoriasis and atopic dermatitis during the COVID-19 pandemic. Dermatol Ther. 2020;33(5):e13687. doi:10.1111/dth. 13687

5. Chiricozzi A, Talamonti M, De Simone C, et al. Management of patients with atopic dermatitis undergoing systemic therapy during COVID-19 pandemic in Italy: data from the DA-COVID-19 registry. Allergy. 2021;76(6):1813-1824. doi:10.1111/all.14767

6. Ruggiero A, Megna M, Annunziata MC, et al. Teledermatology for acne during COVID-19: high patients' satisfaction in spite of the emergency. J Eur Acad Dermatol Venereol. 2020;34(11):e662-e663. doi: $10.1111 /$ jdv. 16746

7. Villani A, Megna M, Scalvenzi M, Fabbrocini G, Ruggiero A. Teledermatology and chronic skin diseases: real life experience in a Southern Italian Dermatologic Centre. Dermatol Ther. 2020;33(6): e13839. doi:10.1111/dth.13839

8. Megna M, Ruggiero A, Marasca C, Fabbrocini G. Biologics for psoriasis patients in the COVID-19 era: more evidence, less fears. $J$ Dermatolog Treat. 2020;31(4):328-329. doi:10.1080/ 09546634.2020.1757605

9. Marasca C, Ruggiero A, Megna M, Annunziata MC, Fabbrocini G. Biologics for patients affected by hidradenitis suppurativa in the COVID-19 era: data from a referral center of Southern Italy. J Dermatolog Treat. 2020;1. doi:10.1080/09546634.2020.1769828.

10. Ferrucci S, Romagnuolo M, Angileri L, Berti E, Tavecchio S. Safety of dupilumab in severe atopic dermatitis and infection of Covid-19: two case reports. J Eur Acad Dermatol Venereol. 2020;34(7):e303e304. doi:10.1111/jdv.16527

11. Caroppo F, Biolo G, Belloni Fortina A. SARS-CoV-2 asymptomatic infection in a patient under treatment with dupilumab. $J$ Eur Acad Dermatol Venereol. 2020;34(8):e368. doi:10.1111/jdv.16619

12. Rossi M, Rovati C, Arisi M, Soglia S, Calzavara-Pinton P. Management of adult patients with severe atopic dermatitis treated with dupilumab during COVID-19 pandemic: a single-center real-life experience. Dermatol Ther. 2020;33(4):e13765. doi:10.1111/ dth. 13765

13. Ordóñez-Rubiano MF, Campo I, Casas M. Dupilumab in atopic dermatitis, a protocol for SARS-COV-2-infected patients. Dermatol Ther. 2020;33(6):e14172. doi:10.1111/dth.14172

14. Ordóñez-Rubiano MF, Rubiano-Mojica PC, Young M. Casas HIV-positive male patient with severe atopic dermatitis on dupilumab and SARS-CoV-2 infection, a pioneer hypothesis. Int $J$ Dermatol. 2021;60(4):514-515. doi:10.1111/ijd.15499

15. Carugno A, Raponi F, Locatelli AG, et al. No evidence of increased risk for Coronavirus Disease 2019 (COVID-19) in patients treated with Dupilumab for atopic dermatitis in a high-epidemic area Bergamo, Lombardy, Italy. J Eur Acad Dermatol Venereol. 2020;34 (9):e433-e434. doi:10.1111/jdv.16552

16. Napolitano M, Patruno C, Ruggiero A, Nocerino M, Fabbrocini G. Safety of dupilumab in atopic patients during COVID-19 outbreak. J Dermatolog Treat. 2020;1-2. doi:10.1080/09546634.2020.1771257

17. Georgakopoulos JR, Yeung J. Patient-driven discontinuation of dupilumab during the COVID-19 pandemic in two academic hospital clinics at the University of Toronto. J Cutan Med Surg. 2020;24 (4):422-423. doi:10.1177/1203475420930223
18. Tanabe N, Matsumoto H, Hamada S, Ito I, Hirai T. Dupilumab maintenance therapy in an asthmatic patient with coronavirus disease 2019 pneumonia. Allergol Int. 2021;70(2):274-276. doi:10.1016/j. alit.2020.10.005

19. Bhalla A, Mukherjee M, Radford K, et al. Dupilumab, severe asthma airway responses, and SARS-CoV-2 serology. Allergy. 2021;76 (3):957-958. doi:10.1111/all.14534

20. Cento V, Colagrossi L, Nava A, et al. Persistent positivity and fluctuations of SARS-CoV-2 RNA in clinically-recovered COVID-19 patients. $J$ Infect. 2020;81(3):e90-e92. doi:10.1016/j.jinf.2020.06.024

21. Eger K, Hashimoto S, Braunstahl GJ, et al. Poor outcome of SARS-CoV2 infection in patients with severe asthma on biologic therapy. Respir Med. 2020;177:106287. doi:10.1016/j.rmed.2020.106287

22. Förster-Ruhrmann U, Szczepek AJ, Bachert C, Olze H. COVID-19 in a patient with severe chronic rhinosinusitis with nasal polyps during therapy with dupilumab. J Allergy Clin Immunol. 2020;146(1):218220.e2. doi:10.1016/j.jaci.2020.05.005

23. Simpson EL, Bieber T, Guttman-Yassky E, et al. Two Phase 3 trials of dupilumab versus placebo in atopic dermatitis. $N$ Engl $J$ Med. 2016;375(24):2335-2348. doi:10.1056/NEJMoa1610020

24. Blauvelt A, de Bruin-weller M, Gooderham M, et al. Long-term management of moderate-to-severe atopic dermatitis with dupilumab and concomitant topical corticosteroids (LIBERTY AD CHRONOS): a 1-year, randomised, double-blinded, placebo-controlled, phase 3 trial. Lancet. 2017;389(10086):2287-2303. doi:10.1016/S0140-6736(17)31191-1

25. Holt PG, Rudin A, Macaubas C, et al. Development of immunologic memory against tetanus toxoid and pertactin antigens from the diphtheria-tetanus-pertussis vaccine in atopic versus nonatopic children. J Allergy Clin Immunol. 2000;105(6 Pt 1):1117-1122. doi:10.1067/mai.2000.105804

26. Blauvelt A, Simpson EL, Tyring SK, et al. Dupilumab does not affect correlates of vaccine-induced immunity: a randomized, placebo-controlled trial in adults with moderate-to-severe atopic dermatitis. J Am Acad Dermatol. 2019;80(1):158-167.e1. doi:10.1016/j. jaad.2018.07.048

27. Patruno C, Stingeni L, Fabbrocini G, Hansel K, Napolitano M. Dupilumab and COVID-19: what should we expect? Dermatol Ther. 2020;33(4):e13502. doi:10.1111/dth.13502

28. $\mathrm{Li} \mathrm{CK}, \mathrm{Wu} \mathrm{H}$, Yan $\mathrm{H}$, et al. $\mathrm{T}$ cell responses to whole SARS coronavirus in humans. $J$ Immunol. 2008;181(8):5490-5500. doi:10.4049/jimmunol.181.8.5490

29. Donlan AN, Sutherland TE, Marie C, et al. IL-13 is a driver of COVID-19 severity. medRxiv. 2021;2021. doi:10.1101/ 2020.06.18.20134353

30. Marone G, Granata F, Pucino V, et al. The intriguing role of Interleukin 13 in the pathophysiology of asthma. Front Pharmacol. 2019;10:1387. doi:10.3389/fphar.2019.01387

31. Lucas C, Wong P, Klein J, et al. Longitudinal analyses reveal immunological misfiring in severe COVID-19. Nature. 2020;584 (7821):463-469. doi:10.1038/s41586-020-2588-y

32. Kearns DG, Uppal S, Chat VS, Wu JJ. Assessing the risk of dupilumab use for atopic dermatitis during the COVID-19 pandemic. $J$ Am Acad Dermatol. 2020;83(3):e251-e252. doi:10.1016/j.jaad.2020.06.015

33. Worm M, Simpson EL, Thaçi D, et al. Efficacy and safety of multiple dupilumab dose regimens after initial successful treatment in patients with atopic dermatitis: a randomized clinical trial. JAMA Dermatol. 2020;156(2):131-143. doi:10.1001/jamadermatol.2019.3617

34. Kovalenko P, DiCioccio AT, Davis JD, et al. Exploratory population PK analysis of dupilumab, a fully human monoclonal antibody against IL-4R $\alpha$, in atopic dermatitis patients and normal volunteers. CPT Pharmacometrics Syst Pharmacol. 2016;5(11):617-624. doi:10.1002/psp4.12136

35. SECURE-DERM (Surveillance Epidemiology of Coronavirus (COVID-19). Under Research Exclusion) [homepage]; 2021. Available from: http://www.secure-derm.com. Accessed July 5, 2021. 


\section{Publish your work in this journal}

Clinical, Cosmetic and Investigational Dermatology is an international, peer-reviewed, open access, online journal that focuses on the latest clinical and experimental research in all aspects of skin disease and cosmetic interventions. This journal is indexed on CAS.
The manuscript management system is completely online and includes a very quick and fair peer-review system, which is all easy to use. Visit http://www.dovepress.com/testimonials.php to read real quotes from published authors. 